\title{
DOE/PC/C
}

$\mathrm{DOE} / \mathrm{PC} / 90181--\mathrm{TS}$

DE92 015662

Progress Report for Quarter 2: Jan 1, $199 \AA^{2}$ to March 31, 1992

\section{DEVELOPMENT OF A NON-INTRUSIVE PARTICLE TRACING TECHNIQUE FOR GRANULAR CHUTE FLOWS}

\section{Sponsored by the U.S. Department of Energy Contract Number DE-AC22-91PC90181}

New Jersey Institute of Technology

Departments Of Mechanical \& Electrical Engineering

Newark, New Jersey 07102

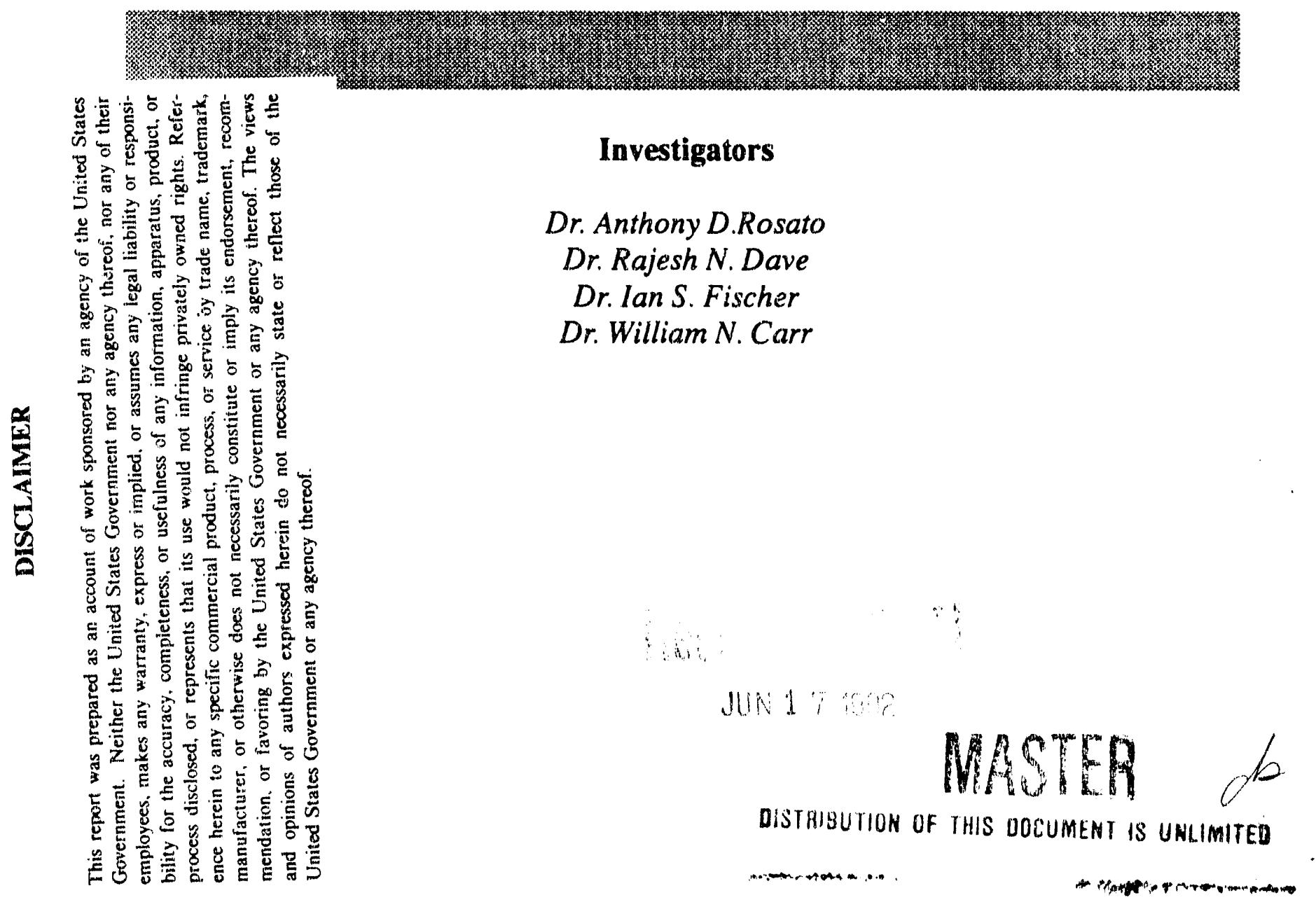


Abstract

I. Introduction

II. Theoretical Model

III. Data Acquisition System 5

$\begin{array}{ll}\text { IV. Electronics } & 6\end{array}$

V. Comparison Between Theory and Experiments 10

VI. Signal Processing 14

VII. Radiosonde Sphere Packaging Design 15

$\begin{array}{ll}\text { VIII. Chute Apparatus } & 17\end{array}$

IX. Related Experiments: Measurement of Particle Properties 19

X. Cluster Analysis of Granular Flows 21

Appendix 1: Chute Drawings, Sphere Packaging Design

Appendx 2: Recent Relevant Papers 


\begin{abstract}
The development of a non-intrusive particle tracking system to follow the trajectory of an individual particle in three dimensions within a mass of particles is necessary to experimentally validate developing theories of inclined chute granular flows in conjunction with particle dynamics models. An understanding of the exact nature of such flows is of critical importance to a variety of industries concerned with solids handling, as well as in natural geological events.

The tracking system, based on the principle of "radiosonde" transmitters coupled to receiving antennae by magnetic induction, is being developed. The radiosonde consists of one or more, orthogonaily placed miniature circuits with integral loop antennas, mounted into a sphere of approximately $3 / 4^{\prime \prime}$ in diameter. The radiosonde sphere position can be traced during the flow down a chute by analyzing the induced voltage signals in the three or more external orthogonal receiving loop antennas due to the transmitter chips.
\end{abstract}




\section{Introduction and Summary of Accomplishments for Quarter}

The objective of this work is to develop an experimental technique to determine the trajectory of a single sphere in a mass of spheres flowing down an inclined chute. A radiosonde transmitter will be developed to be embedded in a prototype test spheres. This transmitter will emit signals which will induce a voltage in parallel sets of receiving antennae via magnetic induction. A theoretical model is developed with which to interpret these voltage signals to the sphere's position.

This report summarizes the progress since this contract work began in January 1991. The reports discusses the model, electronics, and signal processing. Comparisons are shown between the model predictions and experimental data which has been obtained thus far.

New Jersey Institute of Technology is strongly supporting the efforts of the investigators to expand and develop granular flow research within the Mechanical Engineering Division. They have equally matched the DOE support for this con'ract and have provided additional graduate students for other $r$ lated experiments and numerical modeling efforts. This work is briefly discussed in the last sections of this repori.

Below is a list of specific achievements which have been accomplisıled during this reporting period, i.e., January 1, 1992 to March 31, 1992.

\footnotetext{
- New theoretical model with square receiving antennae validated

- Data Acquisition System completed and functional

- Miniature transmitter package with one coil built to fit into sphere

- Tests to compare experimental 1-D trajectory with model-predicted trajectory completed and successful.

- Prototype sphere packaging design completed

- Software to compute the translation and angular velocities of two colliding spheres for subsequent analysis completed.
} 
The use of a circular loop on a rectangular shaped chute results in a "dead space" and a resulting reduction in the signal to noise ratio (See Fig. 1). Therefore a new model was developed for a rectangular shaped receiving loop which could follow the chute's contour very closely.

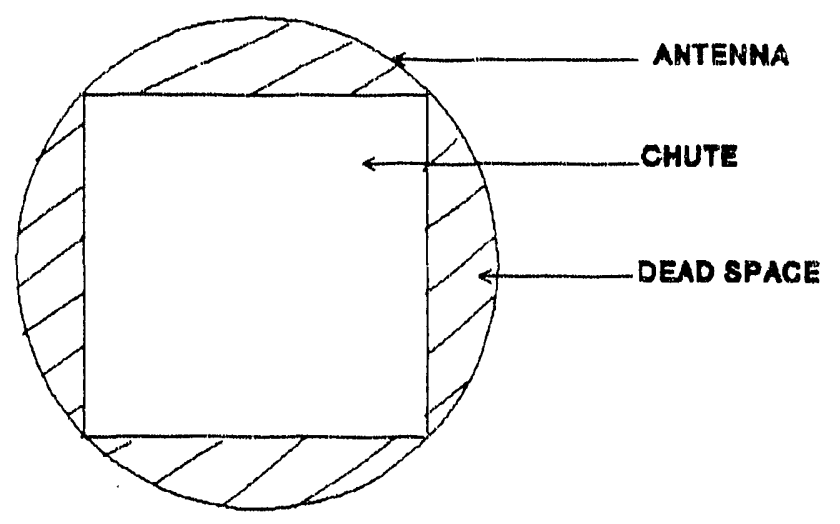

Figure 1: Diagram of "dead space" for circular loop antenna

By proper positioning and size adjustments it can be made more sensitive to movements in one direction. From a purely computational point of view, this model requires less computations due to the absence of elliptic integrals which appeared in a previous model. The model gives the transfer function for the voltage induced as a function of the transmitter position with respect to the receiver. To derive the transfer function it was assumed that a current $I$ is flowing in the receiver coil (See Fig 2). We treat the rectangular loop as four independent filament sources and then apply Superposition theorem to get the composite response by summing up the individual responses. The magnetic field density due to the current $I$ at a position $(x, y, z)$ is given by

$$
\bar{B}=\Sigma_{i=1}^{4}\left(\frac{\left(\mu_{0} I\right)}{4 \pi R_{i}}\left(\cos \phi_{i 1}-\cos \phi_{i 2}\right)\right) \bar{\theta}_{i}
$$

where $\cos \phi_{\mathrm{j}}, R_{i}$ and the position vector $\theta_{\mathrm{j}}$ are functions of the position $(x, y, z)$ and the loop dimensions $l$ and $a$. The magnetic field density vector $\bar{B}$ can now be represented as

$$
\bar{B}=B_{x} \bar{X}+B_{y} \bar{Y}+B_{z} \bar{Z}
$$

The voltage induced in a transmitter coil positioned at $(x, y, z)$ and of area $A$, which is assumed to be elemental, ( i.e. , the magnetic field density does not change over it), is given by,

$$
V=-\omega(\bar{B} \cdot \bar{A})
$$

where $\omega=2 \pi f, f$ being the operating frequency. If the normal to the plane of the transmitting coil 


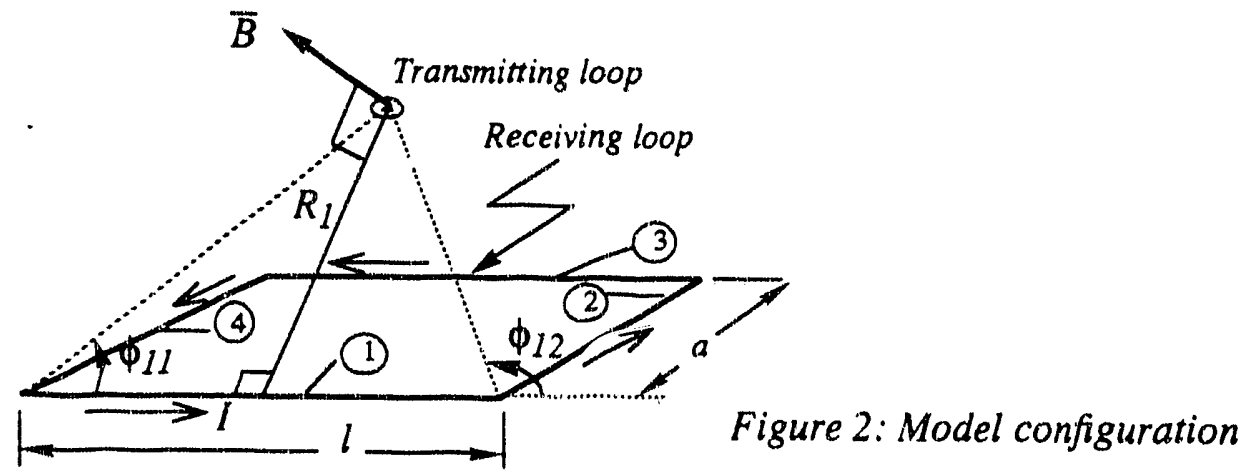

has direction cosines $l, m$, and $n$ respectively, then the induced voltage can be written as,

$$
V=-\omega A\left[B_{x} l+B_{y} m+B_{z} n\right]
$$

An application of the principle of Reciprocity [1], which states that the source and the response in a linear network can be interchanged, gives that a current $I$ flowing in the transmitter will induce a voltage $V$ in the receiver given by the transfer function derived.

\section{Data Acquisition System}

The data acquisition system is the link between the signal processed (amplified and detected) receiver outputs and the reverse simulation program to determine the position using the theoretical model. . Its main parts consist of an IBM compatible 486 PC, the National Labwindows software and the National AT-MIO-16 board (See Fig. 3).

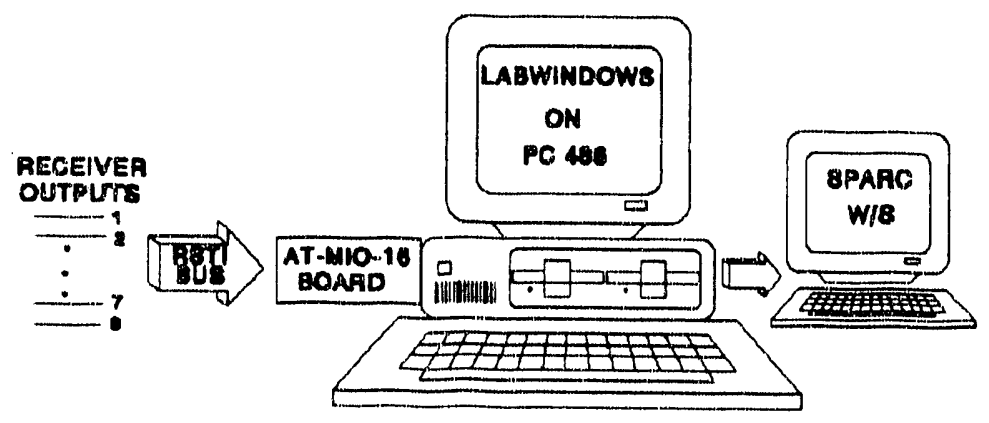

Figure 3: Data Acquisition System

The AT-MIO-16 is a multi function analog, digital, and timing I/O board for IBM PC AT and compatibles. It contains a twelve bit analog to digital convertors (ADC) with upto sixteen analog inputs. It has a $9 \mu \mathrm{s}$ data acquisition convertor which guarantees rates upto $91 \mathrm{~K}$ samples $/ \mathrm{sec}$.

There is a 16 word analog to digital first in first out (FIFO) buffer for fast data acquisitions. The AT-MIO-16 is interfaced to the National Instruments RSTI bus. In the setup, the AT-MIO-16 is 
being used with Lab Windows, a program development software package for test and measurement applications. Lab Windows uses and enhances Microsoft QuickBasic and C with an interactive development environment, function panels to generate the source code, and libraries for data acquisition, instrument control, data analysis, and presentation. The $A D C$ is a 12 bit successive approximation $\mathrm{ADC}$. The 12 bit resolution allows the convertor to resolve its input range into 4096 different steps. All the receiver channels are scanned every $0.1 \mathrm{~ms}$ with a single scan taking $0.1 \mathrm{~ms}$ to get information from all of the channels. The input multiplexer consists of CMOS ana$\log$ input multiplexers and has 16 input channels. If operated in a differential mosle the number of input channels is reduced to 8 . The multiplexer output is fed to an instrumentation amplifier which in turn is followed by a sample and hold circuit and the 12 bit ADC. The output of the ADC is stored on the IBM PC hard disk in an ASCII file which is then input to the "reverse simulation program for position determination".

\section{Electronics}

The "radiosonde" system consists of a transmitter located in the sphere to be tracked, six receiving antennae loops and the associated amplifiers with signal processing. Two antennae are located in each of the orthogonal planes and are placed on opposite sides of the chute. Each antennae pair gives primarily, the displacement of the sphere in the direction as shown in Figures $4 a, b$. This displacement is along a line normal to the planes of the antennae. For this system to function correctly, the currents flowing in the antennae must be very small. If this is not the case, the coupling from each antenna to the others would be large and mask the tracking information. With light antenna loading, the signal received by each antenna is only that received by coupling from the sphere being tracked.

Only a few millivolts, at best, are induced into the large antennae loops, which are approximately $3 \mathrm{~m}$ long by $1 \mathrm{~m}$ wide. At this time, the antenna size (of the pair of antennae) along the length of the chute has not been determined. However, based upon calculations and experimental measurements, accurate determinations may be made by using a total of three antennae with one being placed at a center of the chute by using a large area antennae.

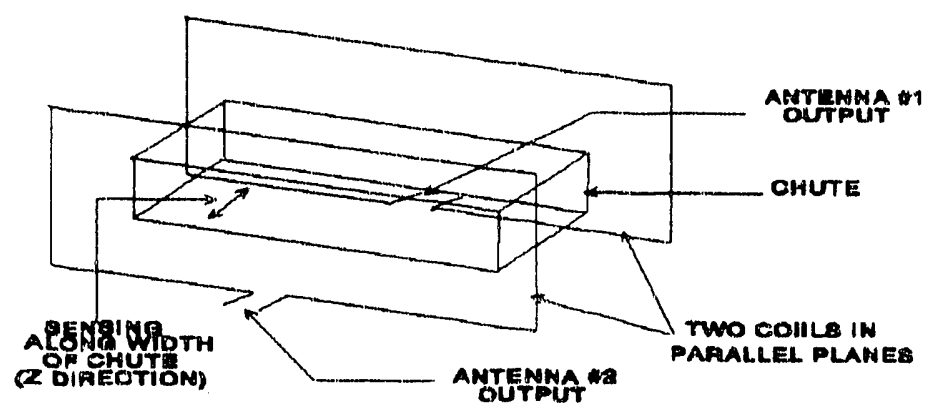

Figure 4a: Sphere location sensing across the chute width 


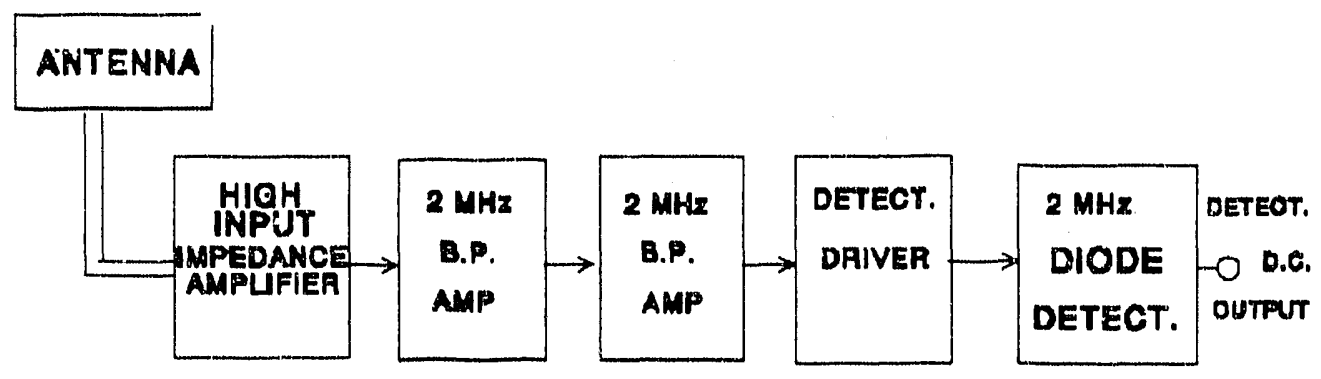

Figure 5: Antenna rf amplified and signal processor block diagram.

The frequency transmitted by the sphere has been tentatively set at $2 \mathrm{MHz}$. A high input impedance circuit is used to couple the antenna signal to a low input impedance bandpass amplifier. This is followed by another bandpass amplifier to further narrow the bandpass and attenuate disturbing, unwanted signals and noise in the $2 \mathrm{MHz}$ region. A low output impedance driver with large signal handling capabilities is used to drive a diode detector. Overall gain from the antenna to the output of the driver is about $46 \mathrm{~dB}$, and the $3 \mathrm{~dB}$ bandwidth is approximately $40 \mathrm{KHz}$. Detecting the rf signal with a diode peak type detector is non-linear, especially when the driving signal is less than one volt peak to peak. Also, the detector is very sensitive to transient spikes due to turn on/off of nearby equipments, since it tends to give an output close to the peak amplitude of the input signal.

Figure 6 shows the block diagram of a balanced demodulator, which gives a linear output ver-

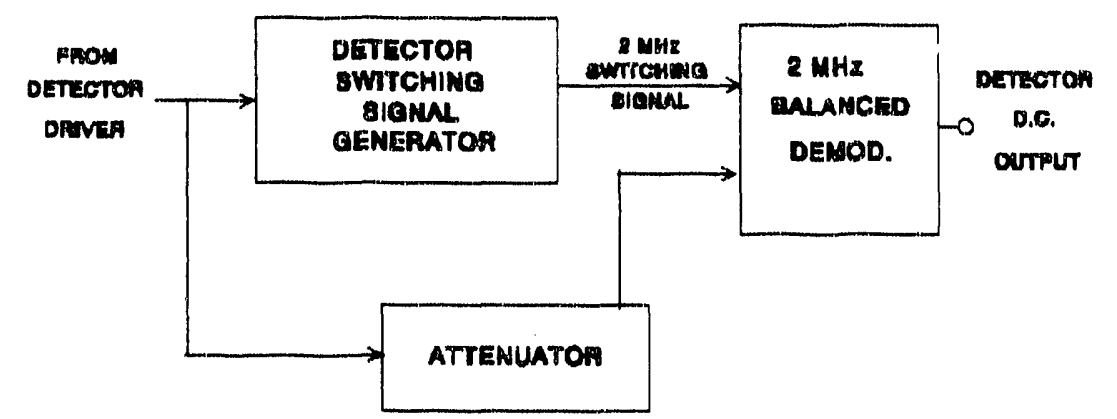

Figure 6: Block diagram of alternative balance demodulator.

sus signal input. However, this requires the generated switching signal to have nearly constant amplitude even when the signal input is at a low level. At low enough signal levels the switching signals will decrease and the detected ouput will be closed to square law rather than linear. In actual operation, linearity at the low sr signal levels for the demodulator will be superior to the diode detectors. The balanced demodulator circuit design and construction has been completed and a schematic of the amplifier and the detectors are shown in Figures 7 and 8. 


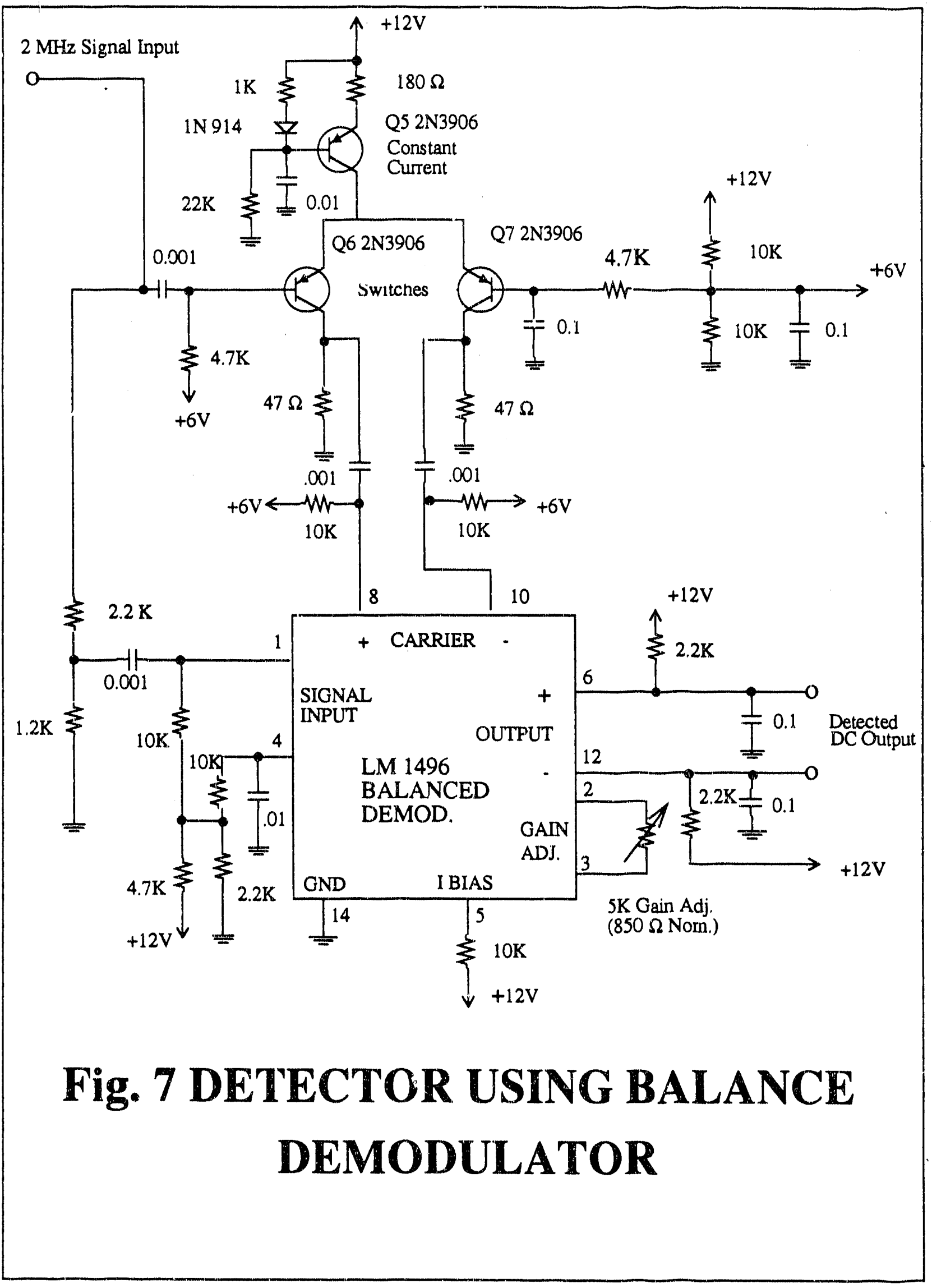




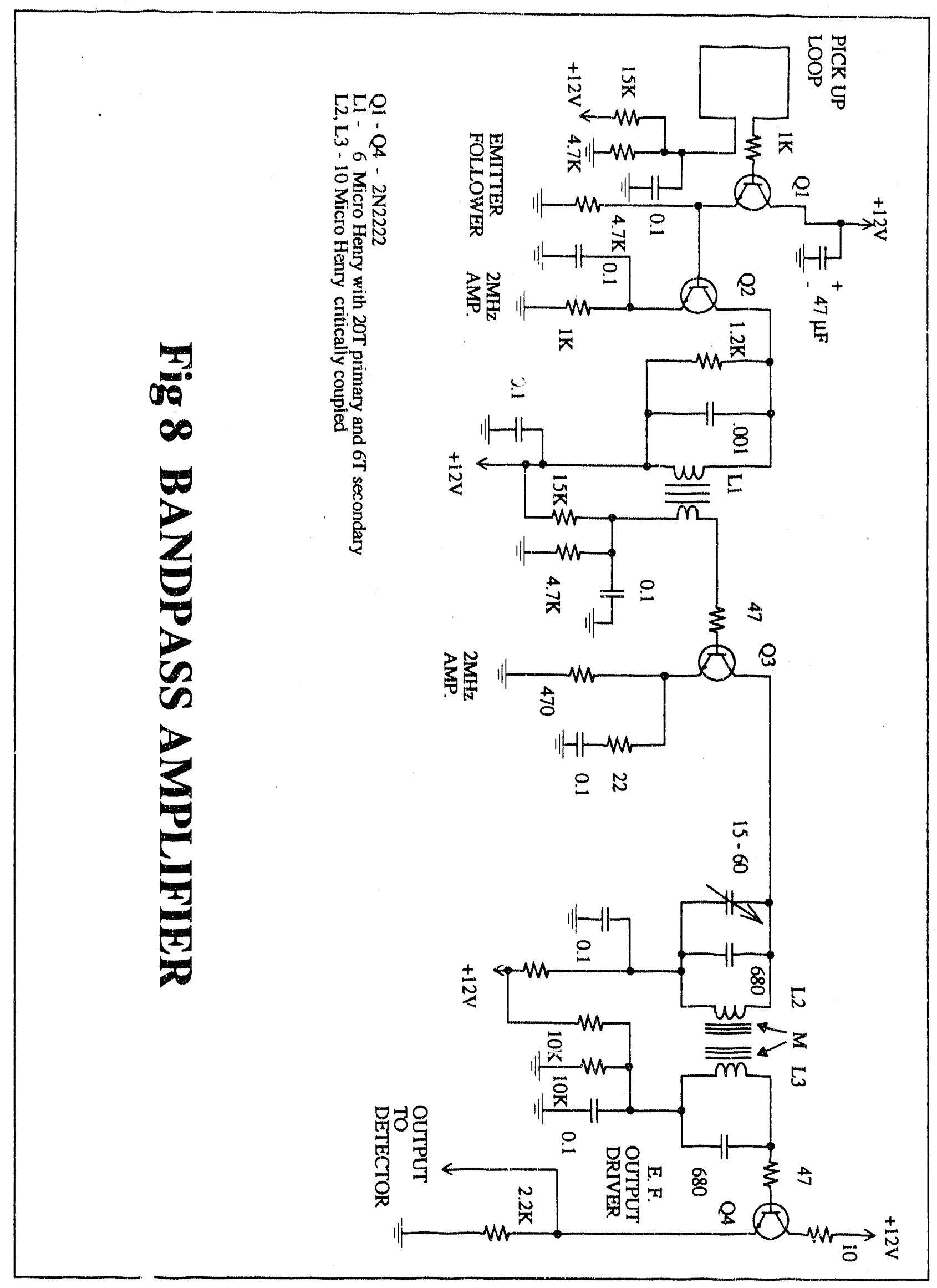


In the sphere which is to be tracked, a simple one transistor oscillator at $2 \mathrm{MHz}$ has been designed and constructed. The same $\mathrm{d} r$ sign has been implemented using surface mounted components on an epoxy printed circuit board. The oscillator operates at $3 \mathrm{~V}$ from the wrist watch cells and draws about $2.8 \mathrm{~mA}$ of battery current. Figure 9 shows the approximate dimensions of the transmitter. A circuit diagram of the oscillator is shown in Figure 10. Feedback to maintain oscillations is supplied by a secondary wound directly over the primary and properly phased. Using a secondary winding increases the efficiency of the oscillation since no a.c. power is dissipated in the base biasing resistors. Also the coil is easier to construct since an additional tap on the primary is eliminated by simply winding a rew turns to give the required transistor based drive. All the components are surface mount except for the coil and the IN914 diode which is extremely small.

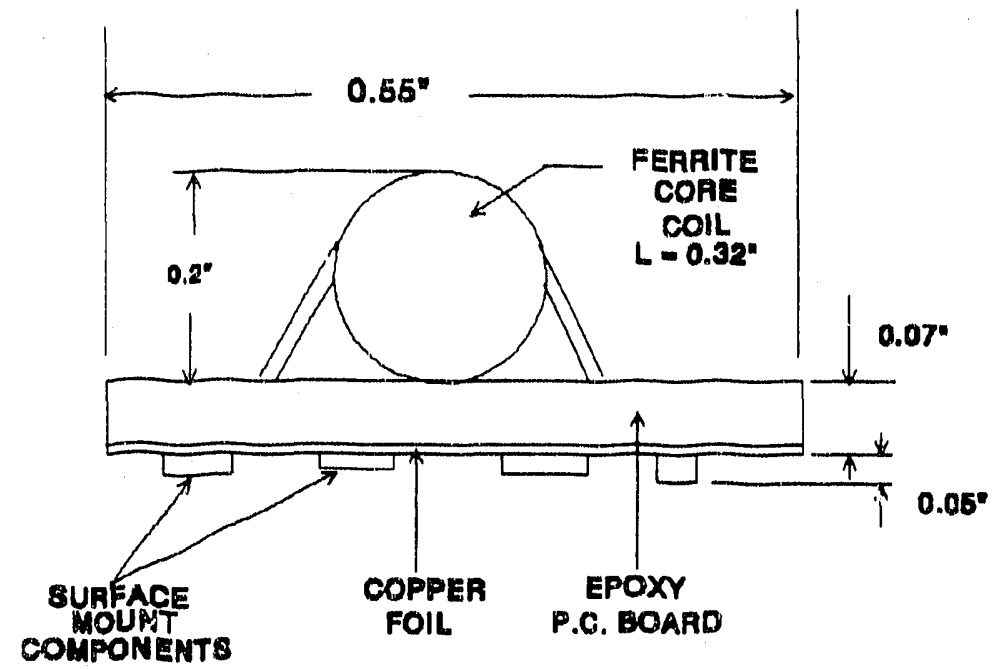

Figure 9 : Approximate dimensions of transmitter electronic circuit

It is possible to use surface mount coils for the oscillator, but the induced voltages into the loop antennae would be too small to be usable. In actual practice, the transmitter coil should be made as large as possible within the constraints of the sphere size requirements. Operation of the oscillator is possible down to $2.3 \mathrm{~V}$ battery supply voltage, which is the entire useful life of the battery.

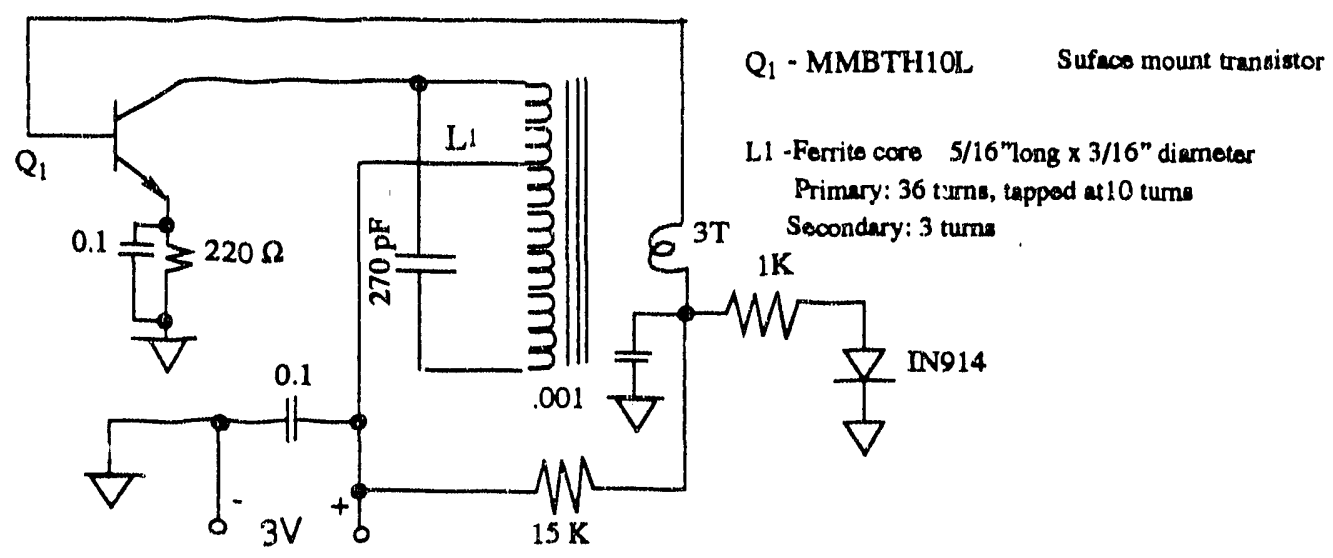

Figure 10 : Hartly Oscillator used as transmitter 


\section{Comparison Between Theory and Experiment}

For a 8" by 4" four-turn receiving antenna, there is a strong correlation between measured and predicted values of voltage in the $Z$ and $X$ directions as shown in Figures 11 and 12, respectively. As noted in the figures, a scale factor was aoplied to the theoretical values. This scaling is a consequence of the fact that the measuring instrument has a $50 \Omega$ impedance to which the receiving antenna was matched. Consequently, an uniform, constant attenuation factor of approximately 20 was found to exist between measured and model predicted values. In Figure 12 , for the $Z=I$ case, it can be seen that both curves exhibit minimas at -6 and 6 and maximas at -4.7 and 4.7. Also, the measured voltage versus coil orientation shown in Figure 13 follows a cosine relation as predicted by the model.

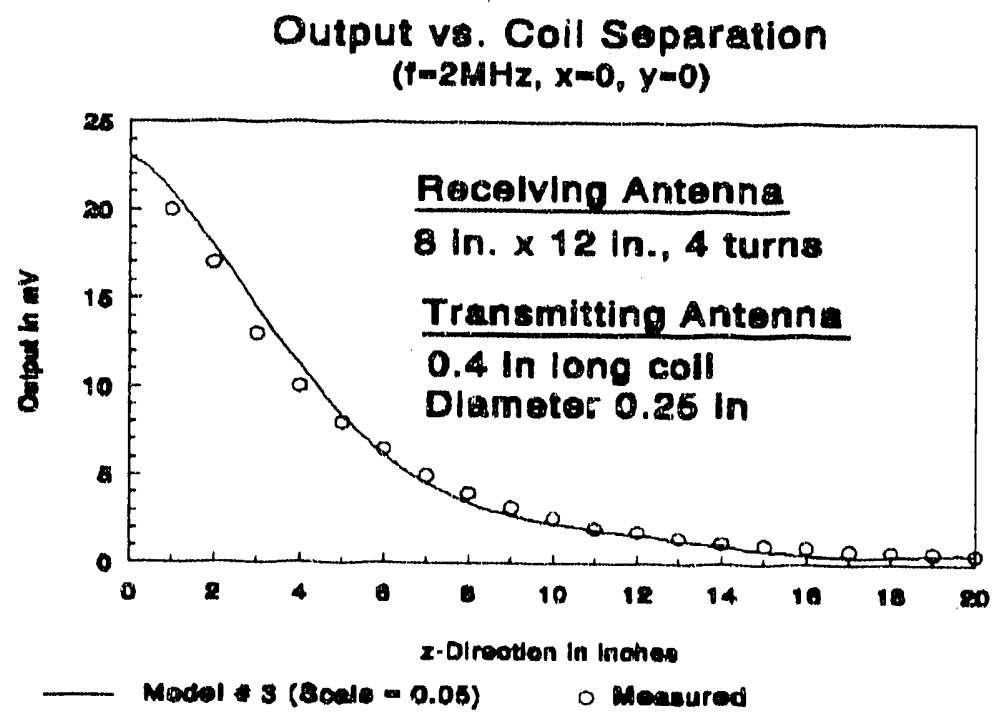

Figure 11 : Output vs. Z Displacement 
Output va. Displacement along $X$-axis $(P=2 M H z, y=0)$

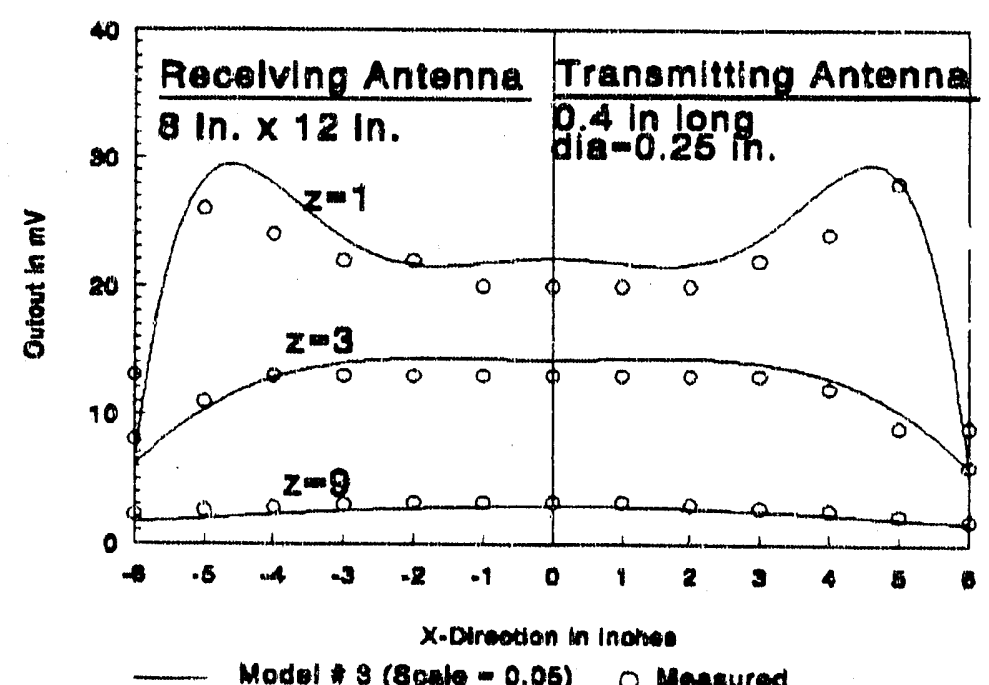

Figure 12 : Output vs. X Displacement

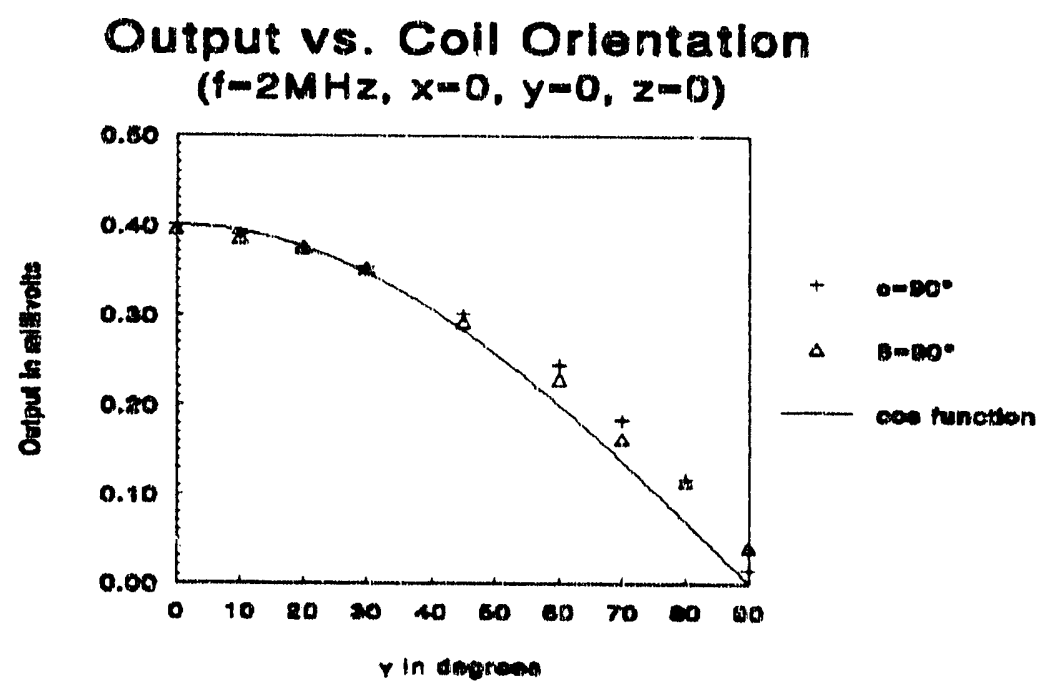

Figure 13 : Output vs. Orientation

The experimental setup for radiosonde sphere tracking consisted of two parallel receiving loops, 120" x 40", separated by 30.2 " as shown in Figures $4 \mathrm{a}$, b. This setup was used for position determination in the $Z$ direction. The transmitter was moved in a path with fixed $X$ and $Y$ pasition values, i.e., only the $Z$ position was varied. The signals picked up from the receiving loops were 
amplified, detected and inputed to the data acquisition system. The data acquisition system stored the measured voltage values in a column form in an ASCII file. This ASCII file was input to the "Reverse simulation program for position determination". Figures 14a,b show the actual position of the coil versus the position predicted using the model. The figure shows a correlation between the two within \pm 1.25 ". The same experiment was then repeated at different $X$ locations.

Predicted Vs. Actual Trajectory at $\mathrm{X}=0^{\prime \prime}$

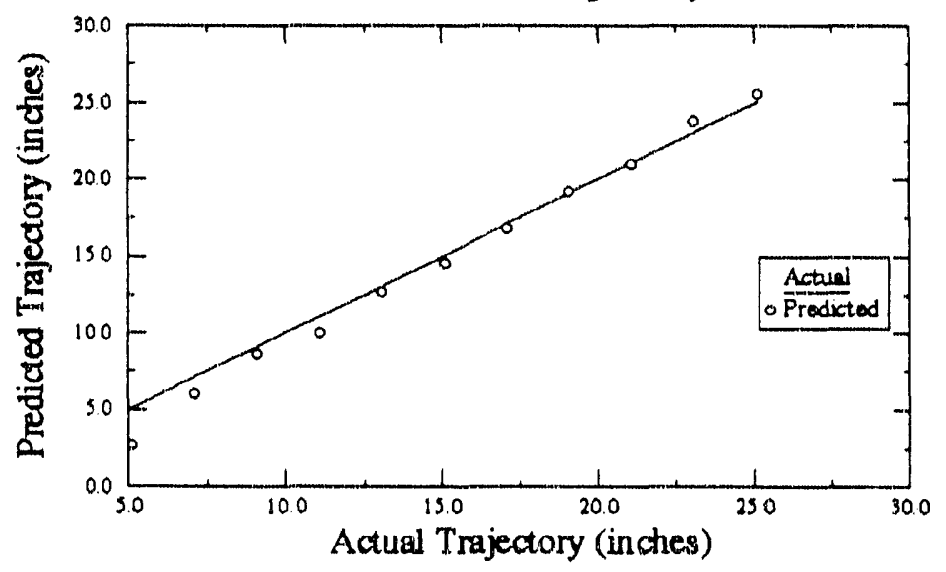

Figure 14a: Predicted vs. Actual Trajectory at $X=0$ "

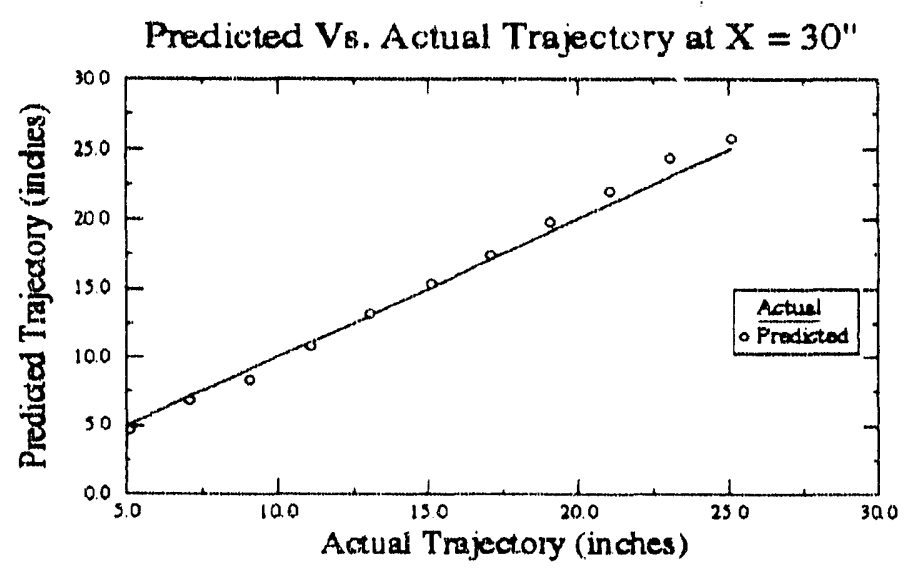

Figure 14b: Predicted vs. Actual Trajectory at $X=30$ inches. 
The technique $t_{1}$ ) determine the position and orientation of the particle from the measured voltages using a mudel that gives the induced voltage transfer function (voltage induced in the receiver as a function of the transmitter location) is presented.

The model presented in Section II results in a system of non-linear equations in six unknorvns for the sphere's position and orientation. A technique to solve an overdetermiried nonlinear system of equations has been developed. This system would result if there were three transmitters within the sphere and at least three receiving antennae. The developed method allows for a unique solution for position and orientation. In the discussion that follows, an outline of the numerical algorithm is presented for the case of nine nonlinear equations in six unknowns. Mathematically, this problem is one of constrained minimization of the cverdetermined, rion-linear system.

The sfhere position and orientation parameters are denoted by $(X)=(x, y, z, \alpha, \beta, \gamma)$, while the voltage indr:ed in the receiver due to the transmitter as predicted by the model is expressed as,

$$
V_{i}(X)=f\left(x_{t r} y_{t r} z_{t r} \alpha_{t r} \beta_{t r} \gamma_{t r}\right) \quad i=1, \ldots 9
$$

The notation above signifies that for each transmitter-receiver pair, there is an associated set of position and orientation parameters, $\left(x_{t n} y_{t r} z_{t r} \alpha_{t r} \beta_{t r} \gamma_{t r}\right)$. The experimentally measured voltage in each receiver is giver $r_{i}$ by $\left(V_{m l}, V_{m 2}, \ldots, V_{m g}\right)$. The $i^{t h}$ residual, $R_{i}(X)$, is then expressed as,

$$
R_{i}(X)=V_{i}(X)-V_{m i} i=1, \ldots 9
$$

The solution of the equation set is conducted by a modification of the non-linear least squares Levenberg-Marquardt algorithm[2]. The problem then is a minimization algorithm, i.e.,

$$
\begin{aligned}
& \text { minimize }\left\|\mathbf{R}\left(\mathbf{X}_{\mathrm{c}}\right)+\mathrm{J}\left(\mathbf{X}_{\mathrm{c}} \cdot \mathbf{X}_{2}\right)\right\|_{2} \\
& \text { subject to }\left\|\mathbf{X}_{+} \cdot \mathbf{X}_{\mathrm{c}}\right\|_{2} \leq \delta_{\mathrm{c}}
\end{aligned}
$$

where $\mathbf{X}_{+}$is the next interate, $\mathbf{X}_{c}$ is the current value, $J\left(\boldsymbol{X}_{c}-X_{2}\right)$ is the Jacobian, and $\delta_{c}$ is the maximum length of a successful step from $X_{c}$, and $\mathbf{R}=\left(R_{1}, R_{2}, \ldots, R_{9}\right)$.

The solution is given by

$$
\mathbf{X}_{+}=\mathbf{X}_{c}-\left(\mathbf{J}\left(\mathbf{X}_{\mathrm{c}}\right)^{\mathrm{T}} \mathbf{J}\left(\mathbf{X}_{\mathrm{c}}\right)+\mathrm{m}_{\mathrm{c}} \mathbf{I}\right)^{-1} \mathbf{J}\left(\mathbf{X}_{\mathrm{c}}\right)^{\mathrm{T}} \mathbf{R}\left(\mathbf{X}_{\mathrm{c}}\right)
$$

The constan's $m_{c}>0$ and $\delta_{c}$ are constants chosen via a scaled trust region method [3] . If $\delta_{\mathrm{c}} \geq\left\|\left(\gamma\left(\mathbf{X}_{\mathrm{c}}\right)^{\mathrm{T}} \mathrm{J}\left(\mathbf{X}_{\mathrm{c}}\right)\right)^{-1} \mathrm{~J}\left(\mathbf{X}_{\mathrm{c}}\right)^{\mathrm{T}} \mathrm{R}\left(\mathbf{X}_{\mathrm{c}}\right)\right\|_{2}$, then $\mathrm{m}_{\mathrm{c}}=0$.

Because of the highly non-linear nature of the functions and the complexity of the solution space the problern of convergence to a global minima is present. Figure 15 shows the residual voltages 
approaching zero as convergence to the global minima is achieved for the case of a single transmitter and six receivers.

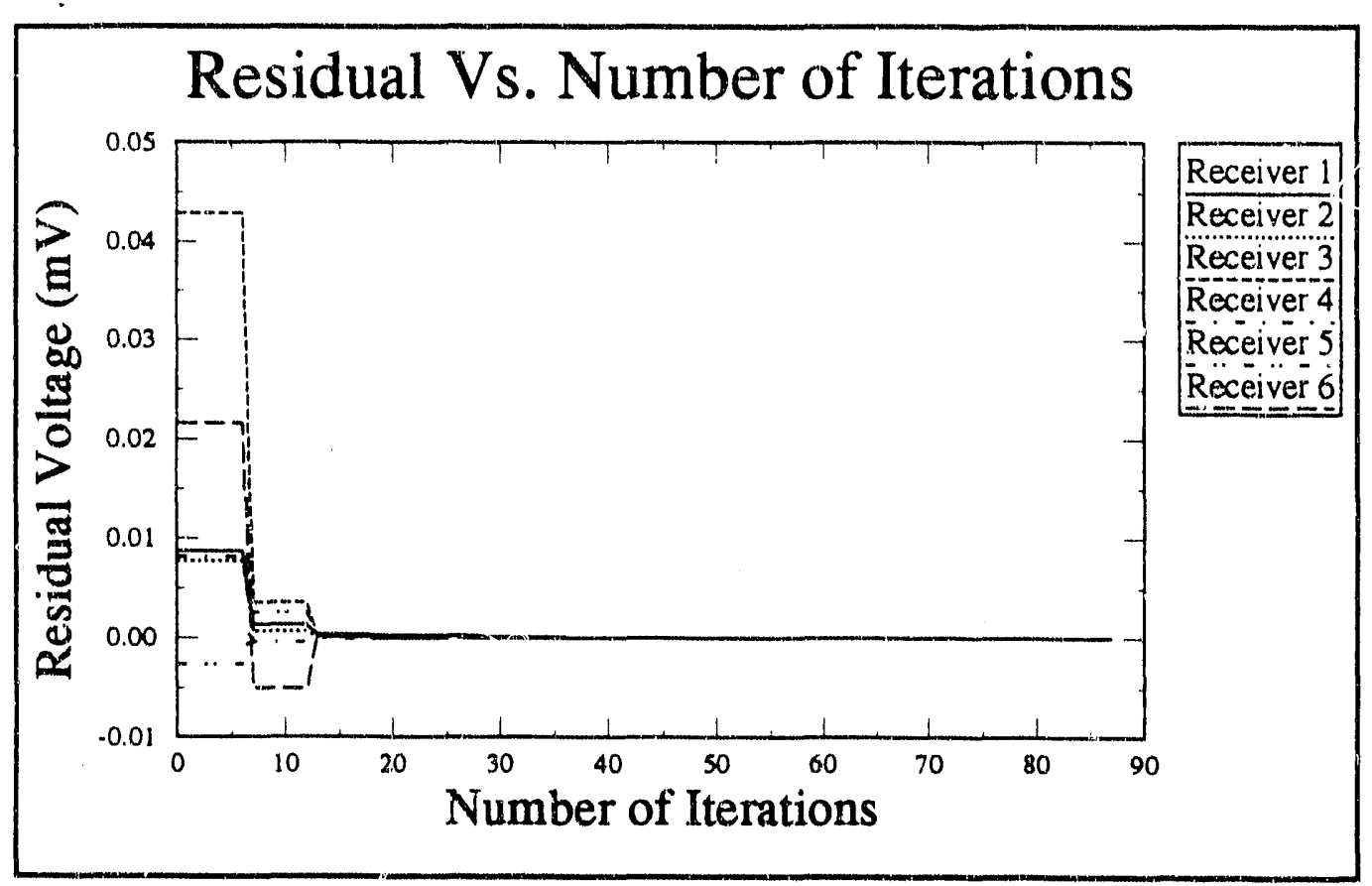

Figure 15: Residual voltages versus number of iterations.

The signal processing task is being conducted in two parts - the Forward Simulation and the Reverse Simulation. The Forward Simulation involves the determination of the voltages indu'ced in the external receivers due to the transmitters based on the model. The Forward Simulation has been completed and it is useful in checking the veracity of the model by comparing the theoretical values with the actual experimantal values. It also provided voltage values neccessary to conduct the Reverse Simulation before the actual experimental values become available.

The Reverse Simulation involves finding the location of the sphere given the experimental voltages. This also has been successfully completed. A simulation program has been written to graphically display the results. In this, the locations of the sphere as determined from the measured voltages would be displayed on two grids as well as in the chute to visualize the trajectory of the particle.

\section{Radiosonde Sphere Packaging Design}

\section{Design Objectives}

1 Good magnetic symmetry.

2 The tracking sphere is statically balance.

3 The tracking sphere has mass similar to the regular sphere.

4 Ease of replacement of batteries, and installation of the transmitter. 
5 Maximum diameter of the tracking sphere is 1 inch.

6 Sphere surface is smooth without any cracks and cavities.

7 Ease of manufacture.

\section{Structure of Design and Size}

The tracking sphere is composed of eight components plus two batteries and a transmitter. In order to easily replace the battery, mount transmitter and manufacture, a structure of two halfspheres with cavities and internal thread 11/16-48 is adopted. A collar with external thread 11/1648 is used to link the two half-spheres together. It is also used to mount and fix the transmitter, weight pad and two battery boxes. The transmitter coil, which is $0.18^{\prime \prime}$ diameter and $0.315^{\prime \prime}$ long , is mounted in the center of the sphere to obtain a symmetrical magnetic field. The transmitter is composed of a print circuit board (PCB) and a coil. The PCB with miniature discrete electronic components is $0.57^{\prime \prime}$ diameter and $0.135^{\prime \prime}$ thick.

Two "371-sliver oxide" batteries provide 3V DC power, and are separately mounted for symmetry. Each battery is $0.175 "$ away from the coil to prevent distortion of the magnetic field. The pair of batteries can be continuously used for approximately 7 hours.

The choice of 1" diameter for the sphere was selected to meet the technical requirements above. The holes in the weight pad and battery boxes are designed to pass the wire connections between the batteries and the transmitter.

\section{Balance Considerations}

1. Static balance: As shown in the figures in the appendx, the center of gravity of the tracking sphere is $(0.0), 0.00,0.00)$ so that the sphere statisfies the requirements of static balance. However, after the real tracking sphere is made, imbalance actually may occur because of the tolerance of manufacturing and assembly.

2. Other properties: The tracking sphere willl be immersed in a flow of other spheres of the same diameter and material as the half-sphere. In order to get the real sphere collision motion trajectory, it is necessary to have the smallest difference of properties between the tracking sphere and other regular spheres. The design provides for the mass and moments of inertia of the tracking sphere to be equivalent to a regular solid sphere, as summarized in the table below.

$\begin{array}{cll} & \text { Tracking Sphere } & \text { Regular } \\ \text { Mass(lbf) } & 0.0228 & 0.0217 \\ I_{x y} & 0.0 & 0.0 \\ I_{y z} & 0.0 & 0.0 \\ I_{x z} & 0.0 & 0.0\end{array}$

The relative error of mass between the tracking sphere and the regular sphere is,

$$
\Delta s=(0.0228-0.0217) / 0.0217=5.07 \%
$$


Because $\left(\mathrm{I}_{\mathrm{xy}}, \mathrm{I}_{\mathrm{yz}}, \mathrm{I}_{\mathrm{xz}}\right)$ are equal to zero, the tracking sphere mass distribution is considered to be balanced. The diameter and position of the wiring holes of the weight pad are designed and revised according to balance requirements.

\section{Material and Density}

All components, except the electric components, are composed of resin material. For the material and density shown in below:

$\begin{array}{lcll}\text { Compronent } & \text { Number } & \text { Material } & \text { Density }\left(\mathbf{l b} / \mathbf{i n}^{\mathbf{3}}\right) \\ \text { half-house } & 2 & \text { nylon } & 0.043 \\ \text { collar } & 1 & \text { nylon } & 0.043 \\ \text { battery box } & 2 & \text { nylon } & 0.043 \\ \text { pad } & 2 & \text { neoprene } & 0.043 \\ \text { weight pad } & 1 & \text { polyester } & 0.060 \\ \text { battery } & 2 & & 0.149^{*} \\ \text { coil } & 1 & & 0.145^{*} \\ \text { PCB } & 1 & & 0.052^{*} \\ \text { *: Actual density } & & & \end{array}$

*: Actual density

\section{Battery Replacement}

Because the tracking sphere is fastened by thread, it is easily opened and closed. After screwing off the half-housing, batteries can be directly removed from the battery boxes. Then fresh batteries will be installed.

\section{Chute Apparatus}

\section{Introduction}

An experiment apparatus has been designed and is in the final stages of construction which can be used for the study of particulate flows down inclined chutes using the radiosonde nonintrusive particle-tracing technique. Drawing of the chute can be found in the Appendix.

\section{Design Objectives}

A chule is being designed with which to conduct tests of the non-intrusive radiosonde particletracing system. There are several design objectives for the chute, all of which will be met in the design. 
- Appropriate Size

The chute has to be sized appropriately for the spheres to be used. Based primarily on the size of available batteries, it is anticipated that spheres as large as $3 / 4$ " diameter will be required. A structure allowing a maximum chute width of 15 " will give a reasonable width to diameter ratio of 20. The available laboratory and standard sizes of construction material indicate a chute length of ten feet, which provides a reasonable length to diameter ratio of 160 . It is anticipated that this length will provide an adequate observation time during which the transition from "at-rest" to "steady-state flow" can occur.

- Radio Transparency

The chute design must facilitate rather than interfere with the experiments. The material of which it is constructed must be "transparent" to the radio transmissions upon which the flow measurements are based. Plastics have the appropriate transmission qualities and therefore will be used instead of metals. Extren fibreglass structural shapes and lucite plate and sheet has been specified, the later being transparent to allow flows to be visually observed as well as making high-speed video-taping possible. It is noted that all of the fasteners employed in the experiment apparatus are plastic, either nylon or Extren polyfibre.

- Adjustability of Inclination angle and chute width

The design will allow the ready adjustment of chute dimensions. A modular construction will allow variation of width and inclination angle.

- Adjustability of Surface Roughness

Provision is provided for substitution of rubber floor mats, styrofoam ceiling panels and textured plastic plates used for lamp diffusers and architectural effects to enable variation of surface roughness and internal damping.

\section{- Simple Fabrication}

The design avoids complex machining. Only sawing and drilling/countersinking holes is required. 


\section{Description}

The chute structure has been designed as several modules. The "chute section" comprises the length of the chute and consists of a 1/2" thick acrylic base plate reinforced by sills of 6 " deep Extren structural shapes. The superstructure of the chute section consists of floor plates and wall plates which can be either smooth acrylic or textured architectural sheets for desired surface roughnesses. Heat-formed acrylic brackets can be located to provide chutes of various widths.

The "low-end support" of the structure is built largely of Extren structural shapes and supports the bottom of the chute section.

"Intermediate supports" of Extren structural shapes wili be constructed if the experiments indicate that additional support is required to provide adequate rigidity and minimize deflection of the chute structure.

The "high-end support" holds the top of the chute, allowing for variation of the inclination angle. To adjust the angle, the end of the chute will be hoisted to approximate position by block and tackle, and then the hinge brackets will be connected to the columns. A jack-screw arrangement will then be used to obtain precise selection of the inclination angle. Arrangement is made for clamping the chute in the exact position required. Construction of the high-end support is largely Extren shapes, with acrylic used for the various details.

\section{Related Experiments: Particle Properties}

The modeling of granular flows and phenomena involving particulates is relevant to many fields, such as industrial solids handling, pharmaceuticals, plastics, materials development and natural granular flows [8]. The highly nonlinear nature of these flows poses enormous complications in developing constitutive models to predict the behavior over a range of conditions. This fact, coupled by the difficulty in performing experiments, has led many to employ particle dynamics simulations to study these flows. Results of the simulation naturally depend on the collisional material properties used, i.e., coefficient of friction and normal and tangential restitution coefficients. In order to better validate developing theories in conjunction with experimental studies, it is necessary to devise a three-dimensional experimental method whereby these properties can be measured.

A prototype experimental apparatus has been built which can lauch two spheres such that their resulting collision will occur at different incident angles. There is no restriction on the intial spin of 
the spheres. The "launching" system and and high-speed digital camera (Kodak EktaPro 1000) will be controlled by a $486 \mathrm{PC}$, which also houses a high-performance image grabber from Imaging Technology. Low level image processing is done using routines from the VISILOG [6] software library.

By performing motion correspondence between a number of points on the sphere (called feature points), three-dimensional motion can be resolved from a single perspective. Low resolution of the camera, however, severly affects this approach. As an alternative, two orthogonal view of the motion are obtained for achieving the best possible accuracy. The measurement of the motion can be divided into translation and rotation. In order to achieve sub-pixel accuracy in translation, special edge detection techniques are used, coupled with boundary detection methods [5]. Measurements of rotation can be achieved through motion correspondence of the set of feature points on the object. The correspondence is achieved using proximal uniformity constraint [9]. The accuracy of the overall procedure is improved using a least squares error approach on an over-determined system of equations.

The specific tasks of the research are as follows:

- Establish the interfact between the PC, the EktaPro 1000, and the sphere projectile triggering mechanism. An IO card from Data Translation (DT2817) is used to trigger the projectiles and recording on the EktaPro. This interface includes the ability to transfer images from the camera to the PC.

- Design and build the experimental apparatus, lighting system and the imagers.

- Calibrate the images to the world coordinate system.

- Perform the collision experiments.

- Transfer image data to the PC, several images at a time.

- Analyze the images to find positions of the spheres and determine the impact time.

- $\quad$ Analyze a set of frames jusi vefore and after the collision.

- Compute the collision parameters from the results obtained.

- $\quad$ Repeat the experiment for spheres of different sizes.

Appendix 2contains an extended abstract entitled, "Collision Parameter Analysis of Rigid Particles by Computer Vision", which was presented in the Second ASME Region II Graduate Student Technical Conference. (April 12, 1992 at Stevens Institute of Technology).

\section{Cluster Analysis of Granular Flows}

The second PI (Dr. Dave) has recently begun to apply his work on pattern recognition and clustering algorithms to study cluster formations which have been observed in granular flows $[19,20,21,22]$. The objective of Dave's work is to develop automated algorithms to track and formation and break-up of cluster formations in the flows. Basically, cluster analysis is one of the 
basic tools for producing classifications from initially unclassified data. Most clustering algorithms based on optimization techniques tend to seek one or the other type of cluster shapes depending on the distance measurement criteria. This is not possible in the case of a granular flow since one does not know beforehand what the shapes will be. Consequently, it is necessary to make as few assumptions about the data as possible in order to avoid any bias. (Note: Families of clustering algorithms have been reported with different applications in mind [10]. Most clustering algorithms based on optimization techniques tend to seek one or the other type of cluster shapes depending on the distance measurement criteria.) It is difficult, though, to find any one method that will work on all types of clustering problems and that will also be able to discard noise to uncover the true structure. The recent approaches, of $R$. Dave $[5,11-18]$ have been able to address these problems.

Several papers by R. Dave have been included in the Appendix 2 for reference.

\section{REFERENCES}

1 Van Valkenburg, M.E., Network Analysis, pg 258, Prentice-Hall, Englewood Cliffs, New Jersey (1974).

2 Dennis, J.E. and Schnabel, R.B., Numerical Methods for Unconstrained Optimization and Nonlinear Equations, Prentice-Hall (1983).

3 Flistcher, R. and Powell, M.J.D., "A Rapidly Convergent Descent Method for Minimization", Comput.J. 6, 163-168 (1963).

4 Muammar, H.K. and Nixon M., "Tristage Hough Transform Multiple Ellipse Extraction," IEE Proceedings-E, 138, 27-35 (1991).

5 Dave, R.N., "Fuzzy Shell Clustering and Applications to Circle Detection in Digital Images," International Journal of General Systems 16, 343-355 (1990).

6 VISILOG, Release 3.6., Noesis S.A.R.L., (1988).

7 Mullier M., Tuzun U., and Walton, O.R., "A Single-Particle Friction Cell for Measuring Contact Frictional Properties of Granular Materials", Powder Tech., 65, 61-74 (1991).

8 German, R.M., Particle Packing Characteristics, Metal Powder Industries Federation, Princeton, NJ (1989).

9 Rangrajan, K., and M. Shah, "Establishing Motion Correspondence," CVGIP: Image Understanding, 54(1), 56-73 (1991).

10 Bezdek, J., Pattern Recognition with Fuzzy Objective Function Algorithms, Plenum, New York, 1981.

11 R.N. Dave and S.K. Bhamidipati, "Application of the fuzzy-shell Clustering Algorithm to Recognize Circular Shapes in Digital Images", Proceedings of the International Fuzzy Systems Association Congress, Seattle, pp. 238-24 (1989).

12 R. N. Dave and K. J. Patel, "Progressive Fuzzy Clustering Algorithms for Characteristic Shape Recognition", Proceedings of NAFIPS'90, pp 121-124, 1990.

13 R. N. Dave and K. J. Patel, "Fuzzy ellipsoidal-shell clustering algorithm and detection of ellipsoidal shapes", Proceedings of the SPIE Conference on Intelligent Robots and Computer Vision IX: Algorithms and Techniques, Boston, pp. 320-333, Nov. 1990. 
14 R. N. Dave and K. Bhaswan, "Adaptive C-Shells Clustering", Proceedings of the North American Fuzzy Information Processing Society Workshop, Columbia, Missouri, pp. 195199, 1991.

15 R. N. Dave and K. Bhaswan, "New Measures for evaluating fuzzy partitions induced through c-shells clustering", SPIE Conference on Intelligent Robots and Computer Vision X, Boston, November 1991.

16 R. N. Dave air" K. Bhaswan, "Adaptive Fuzzy c-shells clustering and detection of ellipses", submitted to IEEE Trans. Neural Networks.

17 R. N. Dave, "Generalized fuzzy c-shells clustering and detection of circular and elliptical boundaries", to appear in Pattern Recognition.

18 R. N. Dave, "Characterization and detection of noise in clustering", Pattern Recognition Letters, Aug. 1991.

19 Walton, O.R., Kim H.J., Rosato, A.D, "Microstructure and Stress Differences in Shearing Flows," Mechanics Computing in 1990s and Beyond, Proceedings of the ASCE Engineering Mechanics Division, May 20-22, 1991.

20 Hopkins, M.A. and Louge, M.Y., Phys. Fluids A, 3(1), pp $45-57$ (1991).

21 Babic, M., "Particle Clustering: An Instability of Rapid Granular Flows," Proceedings of the Second US-Japan Seminar on Micro-Mechanics of Granular Materials, Elsevier (1992), in press.

22 Sanders, B.E., and Ackermann, N.L., "Microstructure in Rapid Gravity Channel Flow," Proceedings of the Second US-Japan Seminar on Micro-Mechanics of Granular Materials, Elsevier (1992), in press. 
APPENDIX 1

\section{Chute Drawings SpherePackaging Design}




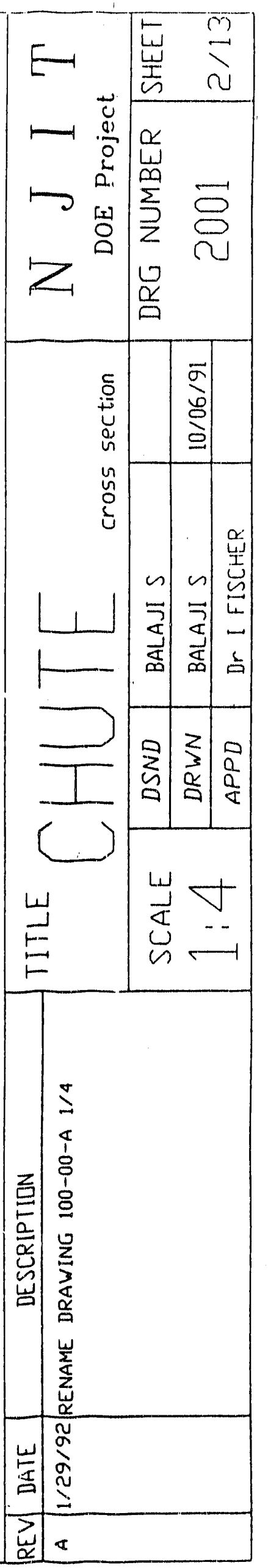



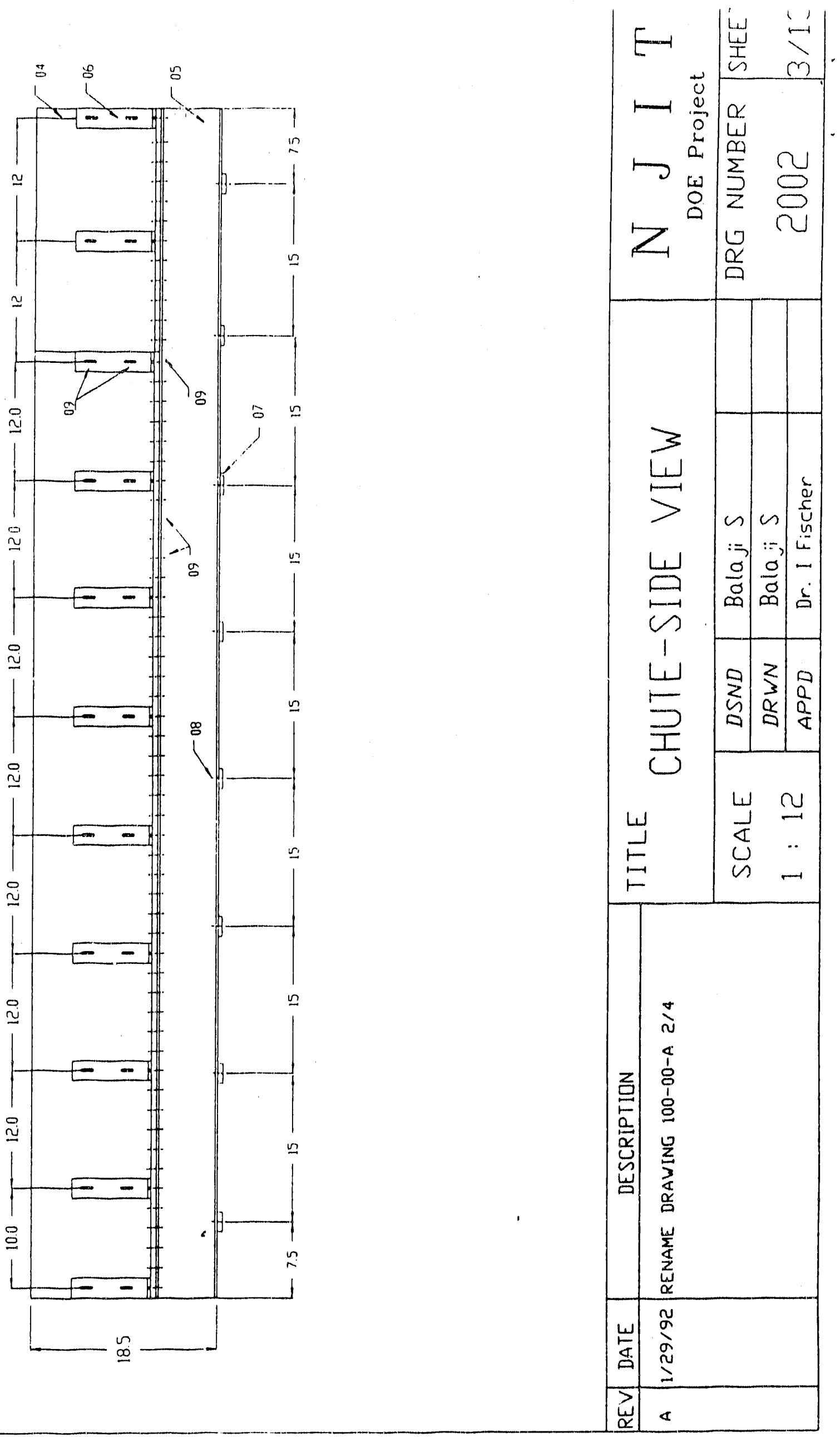

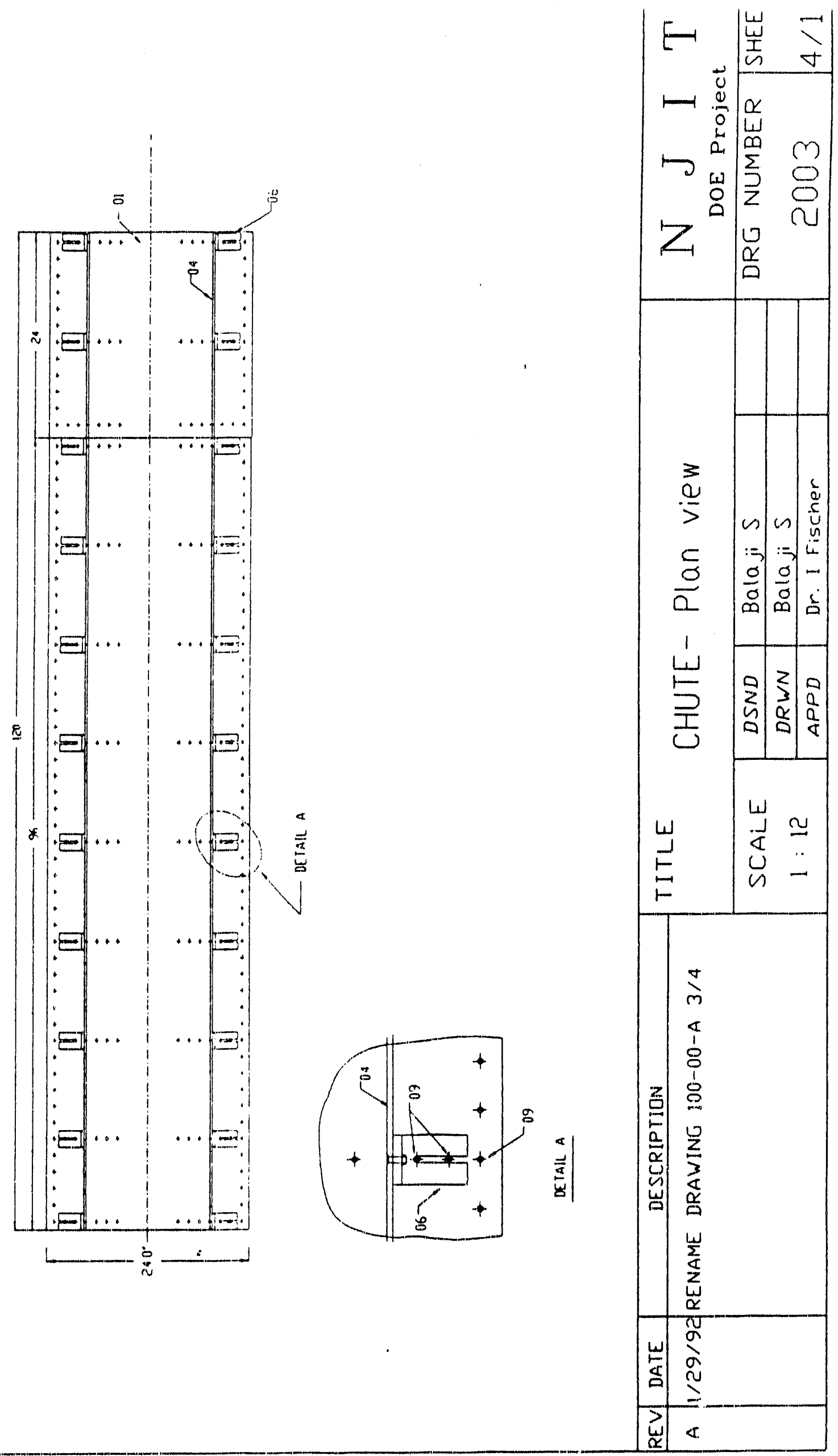

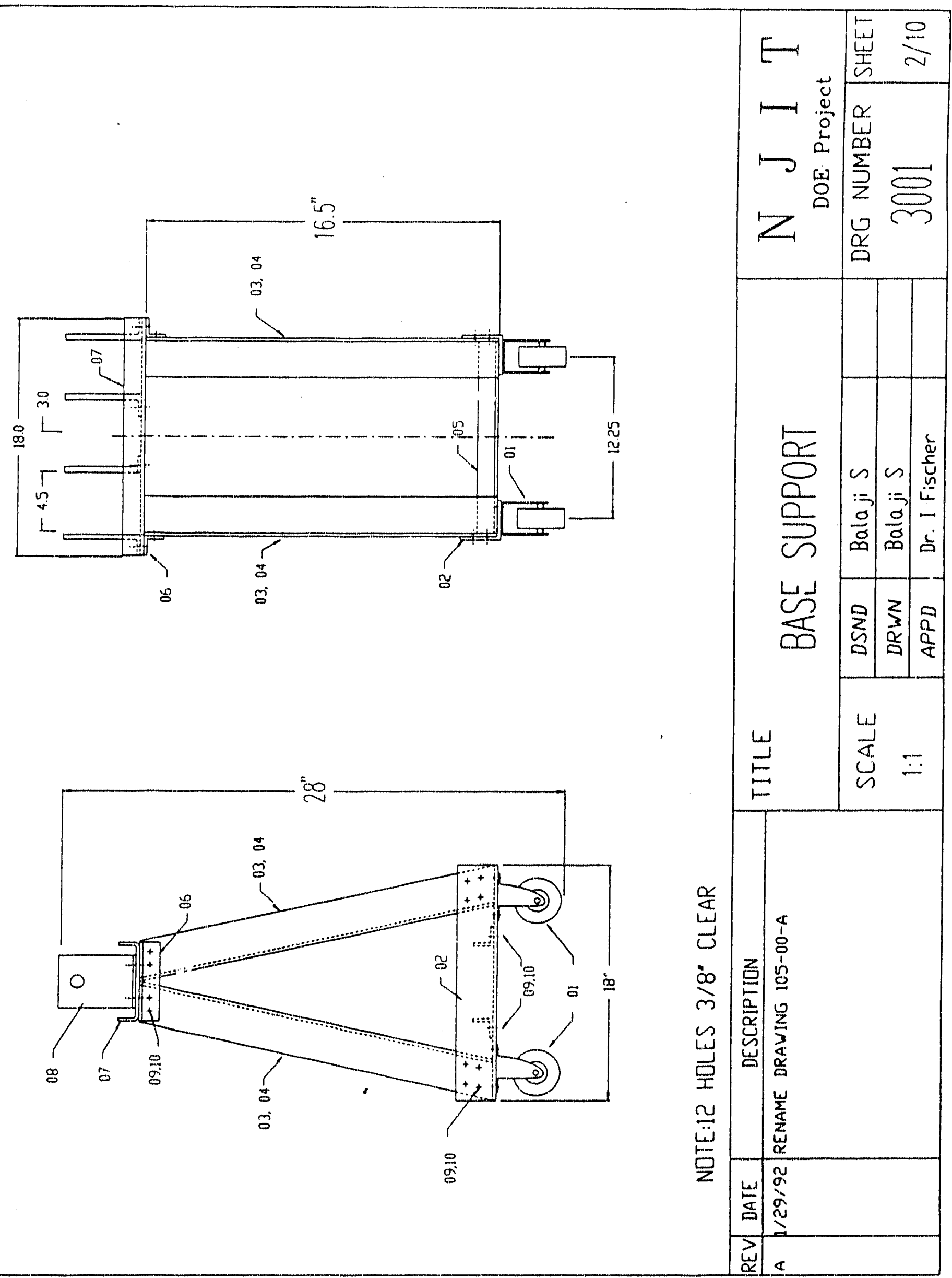


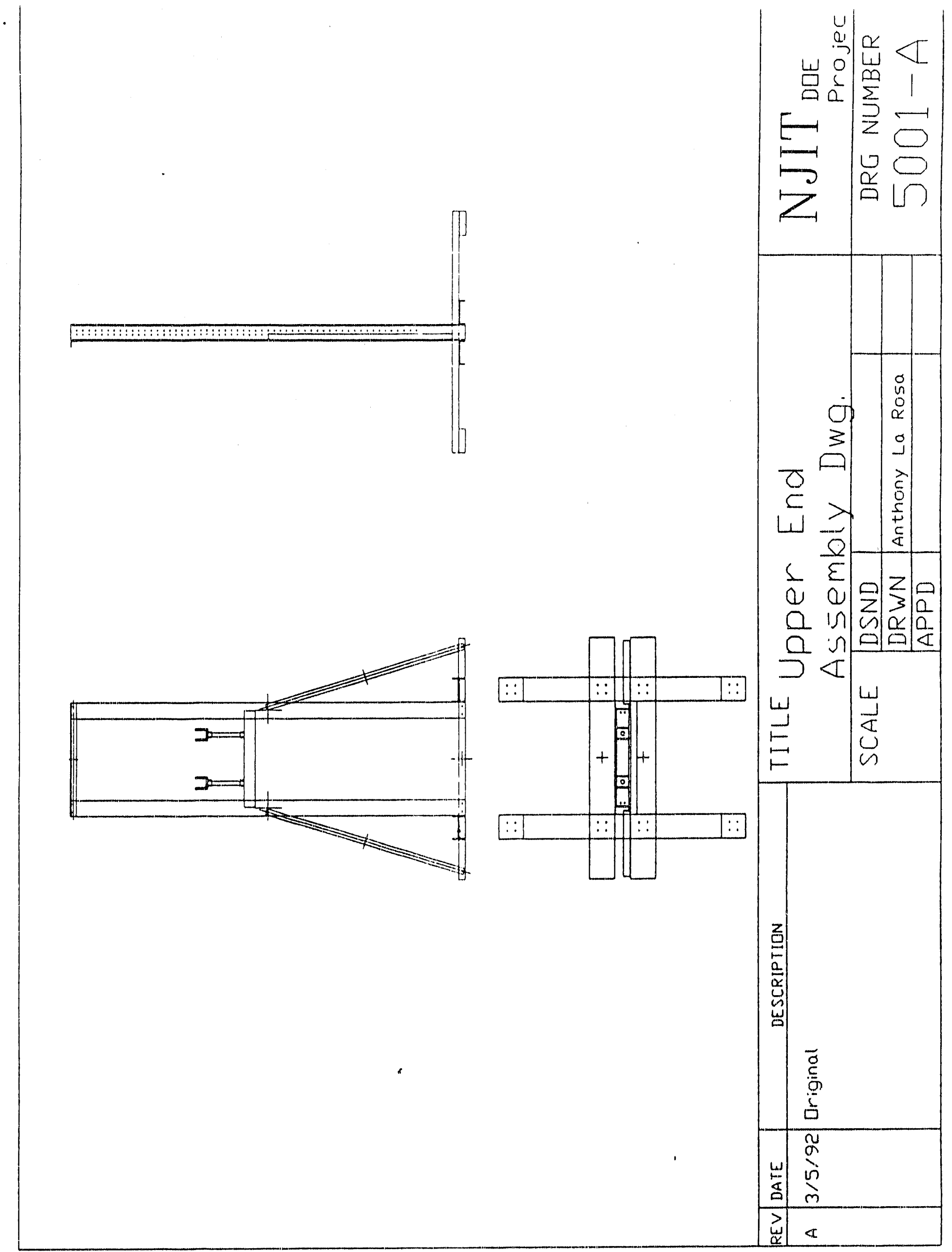




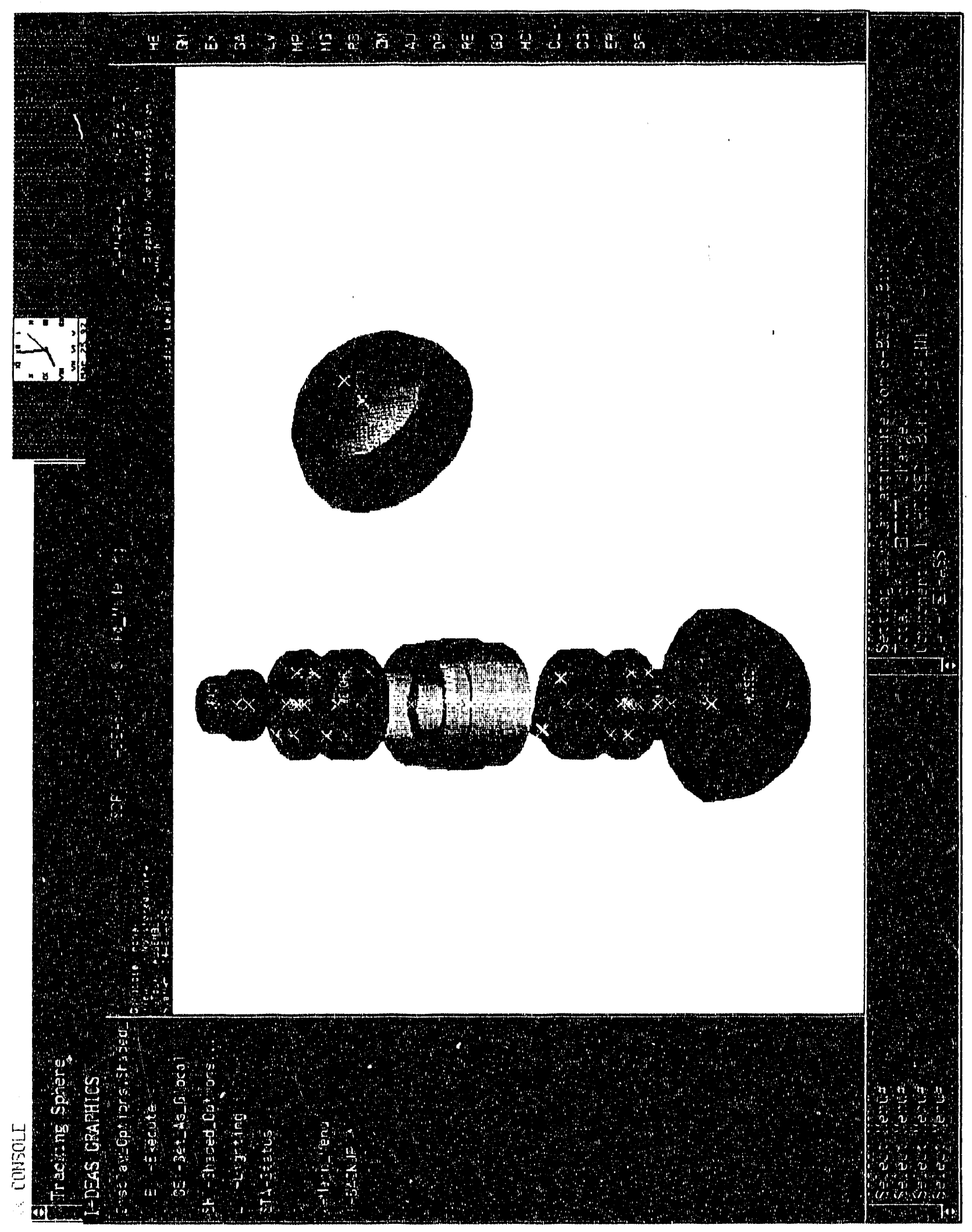




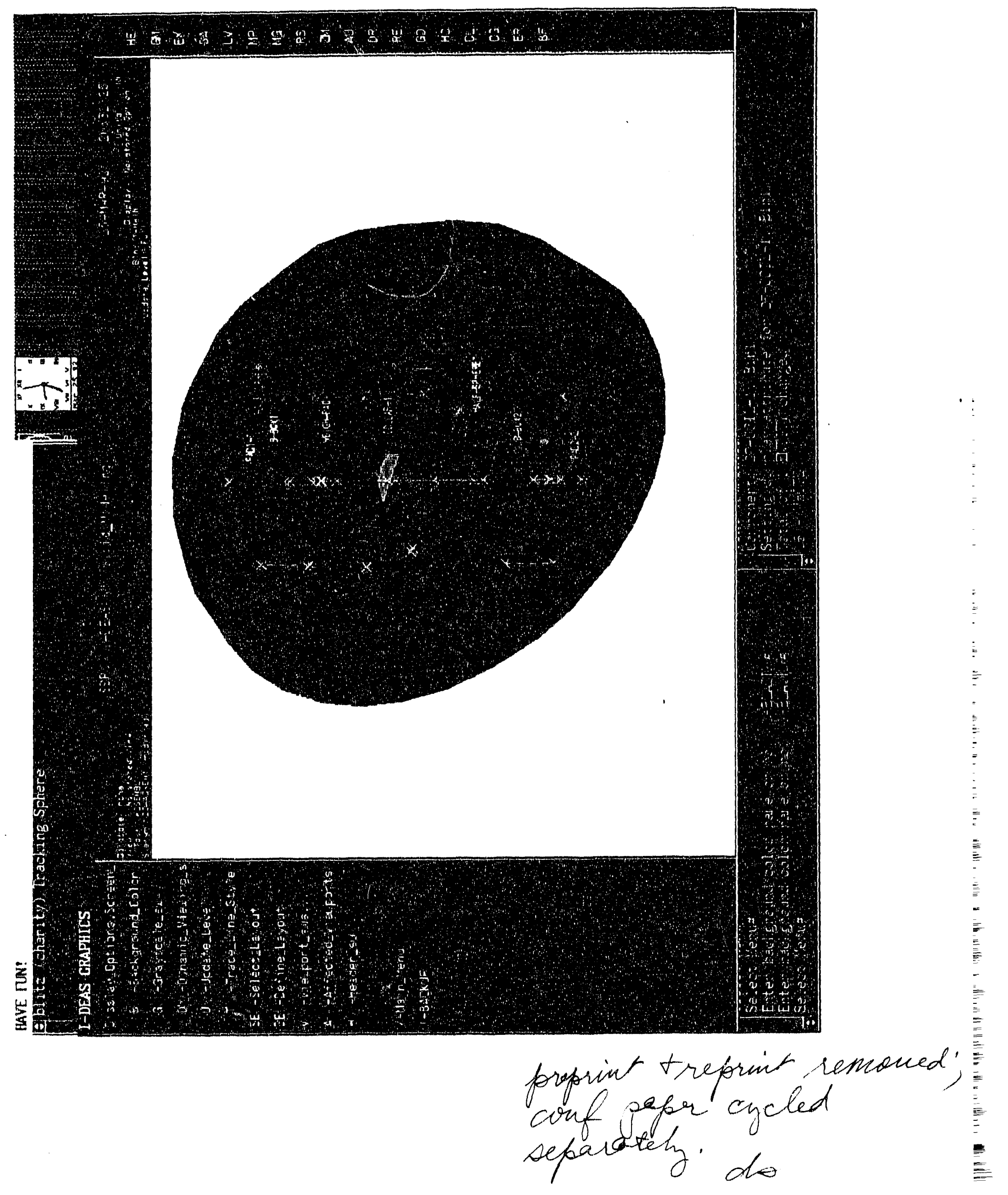



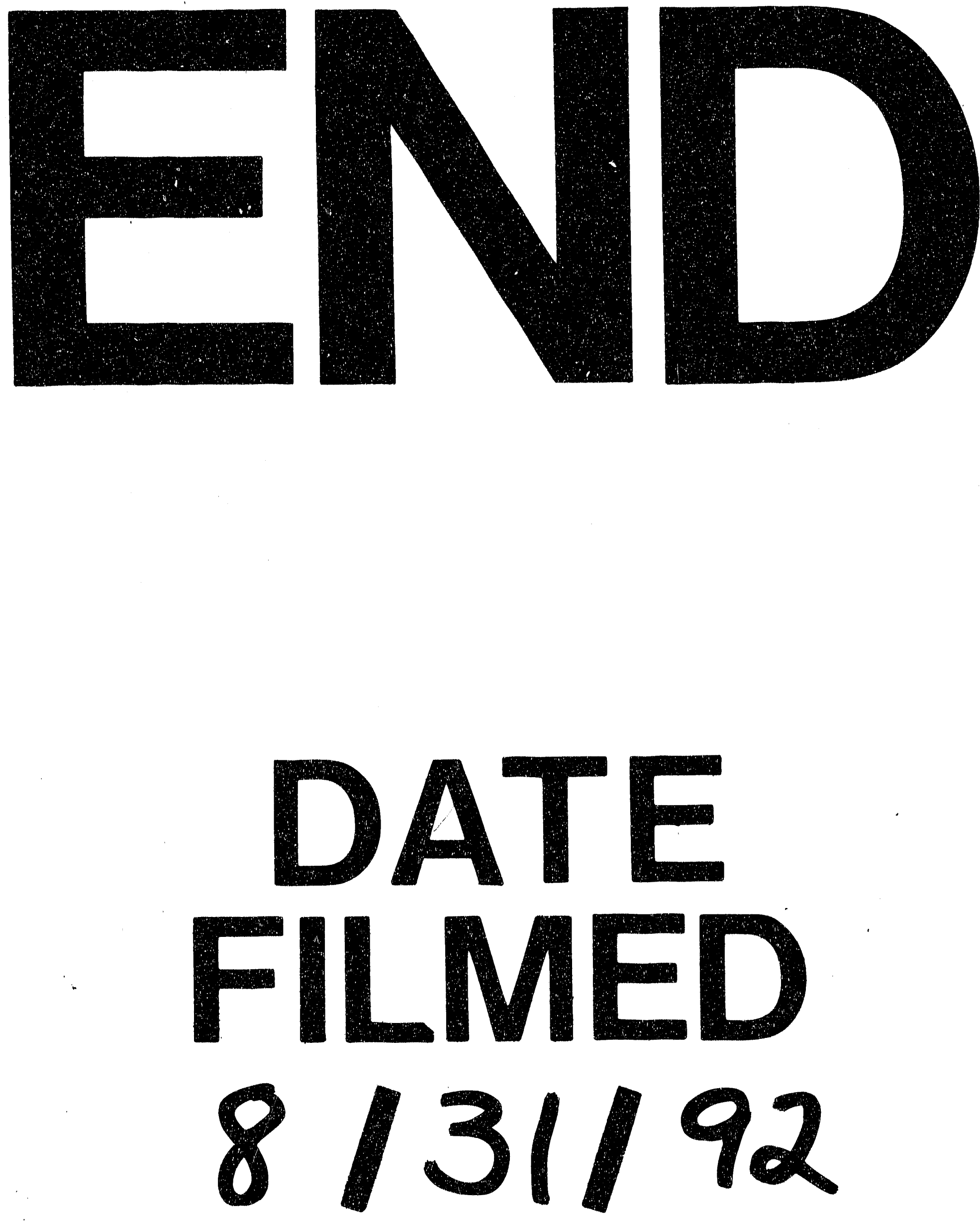

1 
